\title{
Factores de riesgo en personas privadas de libertad en cárceles peruanas por delitos patrimoniales
}

\author{
Risk factors in persons deprived of liberty in Peruvian prisons for property \\ crimes
}

\author{
Marlon Wilbert Florentini Castañeda ${ }^{1}$ \\ Universidad Nacional Federico Villarreal
}

Recibido: $05-04-21$

Aceptado: $12-10-21$

Publicado: $23-12-21$

\begin{abstract}
Resumen
La presente investigación se orienta a explorar los factores de riesgo del contexto personal, familiar y social, presentes en la historia de vida de las personas privadas de libertad, hombres y mujeres sentenciadas por delitos contra el patrimonio. Con tal propósito se diseñó un estudio empírico cuantitativo no experimental, de corte transversal y de alcance descriptivocomparativo. Para la investigación se seleccionó una muestra de reclusos compuesto por sentenciados de los establecimientos penitenciarios de régimen cerrado ordinario Lurigancho y Callao, también una muestra de reclusas del establecimiento penitenciario de Chorrillos comunes, conformando en total 358 personas. El instrumento de recolección de datos se elaboró en base a la ficha psicosocial aplicable por el Instituto Nacional Penitenciario del Perú y fue aplicado mediante entrevista directa con cada integrante de la muestra. Los datos fueron procesados mediante el programa estadístico SPSS. Los resultados muestran que tanto las reclusas como reclusos presentan similares factores de riesgo los contextos individual, familiar, educativo y social a lo largo de su historia de vida. Estos factores deberían ser tomados en cuenta en los programas y políticas de prevención de criminalidad y delincuencia.
\end{abstract}

Palabras clave: Factores de riesgo; delincuencia; delitos contra el patrimonio; privados de libertad; reclusos.

\begin{abstract}
This research is aimed at exploring the risk factors of the personal, family and social context, present in the life history of people deprived of liberty, men and women sentenced for crimes against property. For this purpose, a non-experimental, cross-sectional, quantitative empirical study with a descriptive-comparative scope was designed. For the investigation, a sample of inmates was selected, made up of sentenced persons from the ordinary closed regime prisons Lurigancho and Callao, as well as a sample of inmates from the common Chorrillos
\end{abstract}

1 Docente en segunda especialidad en Universidad Nacional Federico Villarreal, Lima, Perú. Autor para correspondencia: mwflorentini@hotmail.com - fcastaneda@unfv.edu.pe ORCID: https://orcid.org/0000-0001-6463-9653

(C) Los autores. Este artículo es publicado por la Revista de Investigación en Psicología de la Facultad de Psicología, Universidad Nacional Mayor de San Marcos. Este es un artículo de acceso abierto, distribuido bajo los términos de la licencia Creative Commons Atribución 4.0 Internacional (CC BY 4.0) [https://creativecommons.org/licenses/by/4.0/deed.es] que permite el uso, distribución y reproducción en cualquier medio, siempre que la obra original sea debidamente citada de su fuente original. 
penitentiary, making a total of 358 people. The data collection instrument was developed based on the psychosocial file applicable by the National Penitentiary Institute of Peru and was applied by direct interview with each member of the sample. The data were processed using the SPSS statistical program. The results show that both female and male inmates present similar risk factors in individual, family, educational and social contexts throughout their life history. These factors should be taken into account in crime and crime prevention policies and programs.

Keywords: Risk factors; delinquency; crimes against property; deprived of liberty; inmates. 
El comportamiento criminal es complejo y multicausal, por ello sus posibles explicaciones deben ser multifactoriales; $y$, sus intervenciones multifacéticas y multidimensionales. Es de incidencia significativa en muchas sociedades; aflictiva, permanente en el tiempo y de difícil erradicación. La revisión sistemática revela que existe una diversidad de teorías que describen y explican la conducta delictiva. Todas ellas explican segmentos de comportamiento criminal, algunas enfocan características individuales, otras enfatizan variables sociales, otras las situacionales.

La teoría del aprendizaje social parte de la premisa principal que la conducta delictiva se aprende a través de cuatro procesos fundamentales, la asociación diferencial, las definiciones, reforzamiento diferencial y la imitación; tienen aquí un rol protagónico la familia y los grupos de iguales. Desde la teoría del control social, la causa de la criminalidad resulta del debilitamiento de los lazos o vínculos con la sociedad; cuando el individuo carece de arraigo social hacia la familia, la sociedad e instituciones sociales, carece también del control disuasorio necesario, facilitándose el camino hacia la comisión del delito. Desde la perspectiva psicológica, el autocontrol, la frustración, la tensión son elementos que intentan explicar la conducta delictiva. Las teorías integradas por su parte plantean la actuación conjunta de varias teorías en la explicación del delito (Serrano, 2009). Las teorías contemporáneas explican este fenómeno a través de una combinación de mecanismos individuales, sociales y ambientales; ninguna hace uso de una única fuente de factores de riesgo (Redondo, 2015).

La teoría criminológica que más aporta a la identificación de factores de riesgo para conductas delictivas es la criminología del desarrollo y del curso de la vida, al ser dinámicas y no estáticas, consideran que los factores de riesgo pueden ser distintos según el ciclo vital de desarrollo de cada persona. En ese sentido, los estudios realizados utilizando estas teorías aportan mucho a la explicación del inicio, persistencia y desistimiento del comportamiento delictivo a lo largo de la vida. Identifican factores de riesgo explicativos y causales que afectan la probabilidad de ocurrencia de estos comportamientos, así como los efectos de los factores de riesgo y protección durante cada ciclo de vida (Tanner-Smith et al., 2012). Es común observar una perspectiva más sociológica del curso de la vida y más psicológica en la criminología del desarrollo. Pero, ambas estudian a los individuos en trayectorias longitudinales, identificando cambios intraindividuales a lo largo del tiempo, el impacto de eventos vitales y críticos, la importancia del entorno social; y, los caminos, transiciones y puntos de inflexión (McGee y Farrington, 2019).

Los factores de riesgo pueden definirse como aquellas características, variables o peligros que, si están presentes en la vida de un individuo, hacen más probable que este desarrolle una enfermedad, lesión o trastorno. En relación a la delincuencia, los factores de riesgo hacen referencia a aquellas características presentes en la vida de los individuos, que aumentan la probabilidad de incursión en conductas delictivas (Hoge y Vincent, 2015). Las consecuencias probables de los 
factores de riesgo en los individuos van a ser diferenciables en función al tiempo de exposición, ciclo de vida que atraviesa el individuo, intensidad en la presentación del factor, género, entre otras variables individuales y contextuales.

La evidencia científica, hasta ahora, ha posicionado el enfoque de riesgo como un camino prometedor hacia una gestión más eficiente de prevención de la delincuencia, violencia y la reincidencia delictiva, tanto en instituciones cerradas como en la comunidad. Existe consenso respecto al efecto acumulativo de los factores de riesgo, a mayor número, mayor probabilidad de aparición conductas no deseadas; aunque esta relación no es absoluta. Diferentes combinaciones de factores de riesgo contribuyen a diferentes efectos y los riesgos generales del comportamiento antisocial tienden a aumentar exponencialmente dependiendo del número de factores de riesgo (Casey, 2011).

La conducta delictiva no es resultado ni tiene explicación causal a través de un solo factor o un solo proceso, resulta de la combinación de múltiples factores provenientes de diferentes contextos como el individual, familiar, educativo, laboral, social y comunitario. El momento que aparece, su permanencia, su intensidad, el periodo vital del sujeto y las combinaciones, son importantes para diferenciar sus efectos criminógenos, que son mayores ante la ausencia de factores de protección que los amortigüen o neutralicen (Redondo, 2015).

Una fuente importante de factores de riesgo para la conducta delictiva, es el ámbito individual, dentro de ella el consumo de drogas tiene una compleja relación con la delincuencia, no hay evidencia para otorgarle una relación causa y efecto directo, pero sí asociación significativa. El consumo de drogas no conduce en todos los casos a conductas delictivas, son muchos los factores que median este proceso, la pobreza, trastornos de personalidad, factores culturales y sociales, amigos consumidores o contacto con la prisión (Esbec y Echeburúa, 2016). También existen hipótesis que afirman que el consumo de drogas y las conductas delictivas son resultado de la existencia de determinantes comunes, los sujetos expuestos a estos factores pueden desarrollar ambas conductas indistinta o conjuntamente (Otero, 1997).

Torres y Gaytán (2018) encuentra que las adicciones y los comportamientos violentos en el círculo familiar o en el entorno en el que el joven se desarrolla son dos tipos de factores de riesgo frecuentemente correlacionados con conductas violentas. Para Uceda-Maza et al., (2016) si bien la trayectoria delictiva guarda relación significativa con el abuso de sustancias, no es tanto la misma droga que incide en la delincuencia, sino las circunstancias que rodean el escenario vital de consumo y que lo empujan a situaciones adversas.

Otros factores importantes relacionados con la conducta delictiva o violenta posterior son, la edad temprana de inicio de la conducta delictiva, su alta frecuencia y la gravedad del delito (DeLisi y Piquero, 2011). La evidencia es 
recurrente en relación a la edad temprana de inicio y una carrera delictiva más larga (Loeber y Farrington, 2012). Es importante señalar que factores como el ingreso a prisión, consumo de drogas y mantenimiento de amigos con conductas antisociales y delictivas activas, retrasan o dificultan la desistencia delictiva. También la conducta agresiva en la infancia predice la delincuencia posterior.

La posesión y uso de armas en eventos violentos puede revelar dos perspectivas: a) que la motivación del agresor es intensamente violenta, contemplando con antelación el posible daño que podría causar y para lo cual elige el arma apropiada para ejecutar el ataque (Wolfgang, 1958, en Hoskin, 2001); b) que existe un efecto de instrumentalidad del arma, es decir, el acceso y presencia de un arma aumenta la probabilidad de daños independientemente de la motivación del agresor. Las armas pueden potenciar la nocividad al otorgar al agresor poder, coraje y probabilidad de éxito que sin ella no tendría (Zimring, 1968, en Hoskin, 2001).

En el contexto familiar, también se generan múltiples factores de riesgo para la conducta delictiva. Crecer sólo con uno de los padres presenta desventajas en relación a los niños y adolescentes que se encuentran al cuidado de ambos padres o cuidadores. Los hogares biparentales proporcionan mayor supervisión y vigilancia, mientras que la mono parentalidad favorece la probabilidad de delincuencia y victimización, pues existe una persona menos para supervisar el comportamiento de los adolescentes (Felson y Cohen, 1980). Durante la adolescencia, la persona requiere mayor supervisión debido a que incrementa contacto con otros subsistemas externos como amigos y barrio, el tipo de barrio o de amigos con quienes tenga mayor cercanía puede ejercer influencia significativa.

El abandono parental despierta en los adolescentes sentimientos de abandono, soledad y rencor, esta sensación puede iniciar la búsqueda de otras redes sociales donde compensar su insatisfacción (Tenenbaum, 2016). Estrada et al. (2015) encuentra que los hijos criados sólo por la madre, iniciaron de manera más temprana el consumo de alguna sustancia, resaltando la cocaína y el alcohol. En prisión existe gran número de personas que proviene de hogares donde padre y madre, por abandono o trabajo, han estado ausentes en la infancia, en esta realidad algunos de ellos empezaron a delinquir desde temprana edad al crecer bajo la "ley de la calle", sin protección, sin el afecto y ausencia del cuidado de los padres (Lazarte, 2020).

Otro factor de riesgo relacionados con el contexto familiar es la fuga del hogar de niños y adolescentes. Esta acción constituye un escape al estrés cotidiano del espacio familiar caracterizado muchas veces por la conflictividad, negligencia y violencia física, psicológica y/o sexual (Marcelli y Braconnier, 2005). El abandono del hogar es una expresión concreta de la ruptura del adolescente con el sistema familiar. Si la familia orilla al adolescente a la calle, la calle puede orillar al adolescente a la infracción, el término "calle" es un recurso metafórico 
utilizado por los adolescentes para referirse a estar con los amigos y a permanecer frecuentemente en el espacio público. En la calle, la exposición a comportamientos antisociales diversos se incrementa sin la supervisión parental (Tenenbaum, 2016).

Diversos estudios confirman que el maltrato en la niñez, el abuso físico y la negligencia predicen la delincuencia violenta y no violenta posterior (Vidal et al., 2017), aunque con mayor incidencia en hombres, que en mujeres (Alemu et al., 2017). En la adolescencia, el maltrato conlleva a efectos probables, como arrestos, ingresos a prisión, conductas delictivas, conductas violentas, consumo problemático de alcohol, consumo de drogas, conductas sexuales de riesgo, hasta pensamientos suicidas (Thornberry et al., 2010). Los jóvenes que sufren maltrato desde la niñez hasta la adolescencia, presentan más probabilidades de desarrollar conductas antisociales e ingresar al sistema de justicia penal juvenil en relación con aquellos cuyo maltrato familiar se limitó a su niñez (Stewart et al., 2008). La cronicidad del abuso físico incrementa el riesgo de delitos posteriores entre los que fueron abusados, en tanto, no se evidencia un mayor riesgo de delincuencia adulta entre aquellos que sufrieron abuso emocional crónico.

Experiencias familiares no violentas, pero potencialmente traumáticas como la reclusión de los padres y dificultades económicas, pueden tener efectos negativos y duraderos en la salud y bienestar de las personas (Sacks et al., 2014). Es claro entonces que la actividad delictiva de los padres, constituye un factor de riesgo para la realización de conductas antisociales en los hijos (Loeber y Farrington, 2000). Los arrestos por conductas delictivas de familiares cercanos presentan asociación significativa con la delincuencia de los hijos; en estos casos, el pariente con mayor influencia es el padre (Farrington et al., 2001).

También los factores de riesgo del ámbito educativo son importantes, se estima a la educación, específicamente a la escolarización, como un bien que permite conseguir una posición económica y social más elevada en la sociedad (Bazdresch, 2001). La educación secundaria no concluida, viene asociada a mayores desventajas sociales, en términos de empleo y bienestar económico. Para Fachelli (2019), los estudios superiores contribuyen a alcanzar mejores posiciones ocupacionales, incluso mayores que las obtenidas por condiciones familiares. En relación al contexto social, los factores de vulnerabilidad y exclusión social se hallan relacionados con conductas delictivas. Al igual que en el caso de los demás factores de riesgo, a mayor acumulación e intensidad, mayor probabilidad de desarrollar una trayectoria delictiva consolidada (Uceda-Maza y Domínguez, 2017).

Son pocas las investigaciones en Latinoamérica y en el Perú sobre factores de riesgo presentes en la historia de vida de personas que han cometido delitos contra el patrimonio, también es escasa la información sobre estos factores, diferenciando mujeres y hombres. Por tanto, el presente estudio pretende demostrar que hombres y mujeres condenados por delitos contra el patrimonio, presentan múltiples y si- 
milares factores de riesgo personales, familiares y sociales, en su historia de vida. Además, conocer estos factores es importante si tomamos en cuenta que en este tipo delictivo la reincidencia es mayor que en otros tipos de violencia (Capdevila et al., 2014). Conocer estas evidencias son útiles para temas de prevención del delito y para tópicos de tratamiento penitenciario. La prevención desde el enfoque de riesgo implica primero identificar los factores de riesgo clave para las conductas delictivas y sobre ellas implementar acciones de intervención o prevención con el objetivo de disminuirlos o amortiguarlos (Farrington, 2006).

En el sistema penitenciario peruano, las penas tienen función preventiva, protectora y resocializadora; y, es el Instituto Nacional Penitenciario el órgano encargado de su ejecución. La intervención penitenciaria, normativamente, se basa en el enfoque de riesgos, con programas estructurados como Construyendo Rutas de Esperanza y Oportunidad (CREO), dirigido a reclusos primarios por delitos patrimoniales entre 18 y 30 años de edad; programa de Tratamiento de Agresores Sexuales (TAS) y programa para consumidores de drogas. Además, en establecimientos penitenciarios que no cumplen las condiciones mínimas que requieren los programas estructurados, se ejecuta el Programa de Intervención Multidisciplinaria (PIM), que abarca programas especializados para delitos patrimoniales, delitos contra la libertad sexual, delitos contra la vida el cuerpo y la salud, y reclusos de mayor compromiso criminógeno. Todos ellos requieren evidencia sobre los factores de riesgo facilitadores de la conducta delictiva.

\section{MÉTODO}

\section{Participantes}

Participaron 358 personas privadas de libertad procedentes de los establecimientos penitenciarios del Callao, Lurigancho y Chorrillos. En relación a la distribución según sexo, 321 fueron reclusos $(\mathrm{N}=1940)$ y 37 fueron reclusas $(\mathrm{N}=40)$. Los participantes fueron seleccionados mediante muestreo probabilístico estratificado, considerando un valor de heterogeneidad de .5 , margen de error de .05 y nivel de confianza de .95. Los criterios para la inclusión en el marco muestral fueron: a) adultos jóvenes con edades entre 18 y 30 años, b) privados de libertad, c) sentenciados por delitos contra el patrimonio, d) recluidos en los establecimientos penitenciarios de Lurigancho y Callao en caso de hombres; y, e) recluidas en el establecimiento penitenciario de Chorrillos Comunes, en el caso de mujeres.

\section{Instrumento}

Se utilizó un cuestionario con datos de la "Ficha psicosocial para sentenciados" diseñado y utilizado por el sistema penitenciario peruano (Instituto Nacional Penitenciario [INPE], 2018); es parte del documento normativo "Estudio integral y elaboración del programa de tratamiento individualizado del interno. Esta ficha 
reconstruye de manera retrospectiva la historia personal, familiar y social de los reclusos y reclusas. El cuestionario consignó 40 preguntas semiabiertas y fue de aplicación mediante entrevista directa.

\section{Procedimiento}

Se obtuvo permiso del Instituto Nacional Penitenciario para la ejecución de la investigación. La aplicación del instrumento se realizó en cada establecimiento penitenciario donde se encontraban internadas las personas privadas de libertad conformantes de la muestra. Primero se realizó el recojo de datos con los reclusos, en los establecimientos penitenciarios de Lurigancho y Callao; finalmente, se procedió al recojo de datos con las reclusas en el establecimiento penitenciario de mujeres de Chorrillos. Se realizó mediante entrevista directa a cada sujeto y luego de informarles sobre el anonimato de los datos, la confidencialidad y los fines académicos de la información. La participación fue voluntaria, a todos los a los sujetos de la muestra seleccionados de manera probabilística y previo consentimiento informado.

\section{Análisis estadístico}

El análisis de datos se realizó mediante el programa estadístico SPSS, haciendo uso de estadísticos descriptivos para observar frecuencias, promedios y porcentajes en los grupos. Se comparó grupo de hombres y mujeres mediante la prueba chi cuadrada en variables cualitativas, prueba $t$ de student para comparar promedios en caso de variables cuantitativas.

\section{RESULTADOS}

La tabla 1, detalla las características de las muestras del estudio en relación al delito especifico y se obtuvo diferencia significativa en la edad entre reclusos y reclusas $(t=-5.911, p<.001)$.

\section{Tabla 1}

Muestras del estudio

\begin{tabular}{lcccc}
\hline \multirow{2}{*}{ Delitos específicos } & \multicolumn{2}{c}{ Hombres } & \multicolumn{2}{c}{ Mujeres } \\
\cline { 2 - 5 } & Frecuencia & $\mathbf{\%}$ & Frecuencia & \% \\
\hline Estafa & - & - & 2 & 5.4 \\
Extorsión & - & - & 1 & 2.7 \\
Hurto simple/agravado & 17 & 5.3 & 7 & 18.9 \\
Robo agravado & 292 & 91.0 & 26 & 70.3 \\
Robo & 11 & 3.4 & - & - \\
Chantaje & - & - & - & 2.7 \\
No especifica & 1 & 0.3 & & - \\
Edad promedio (años) & 22.95 & & & 25.95 \\
\hline
\end{tabular}




\section{Factores de riesgo relacionados con el individuo}

El 76\% del total de personas del estudio, refirió tener historia de consumo de drogas ilegales. En el caso de reclusos, el porcentaje fue $79.4 \%$ y $51.4 \%$ en caso de reclusas; se evidenció diferencia estadística significativa entre ambos grupos $\left(\mathrm{x}^{2}\right.$ $=-14.575, p=.000)$. No se encontró diferencia significativa $(t=-1.533, p=.127)$ respecto a la edad promedio de inicio de consumo entre reclusos (15.31 años) y reclusas (16.21 años). La primera droga consumida en ambos grupos fue marihuana, representando en total un $92.3 \%$; llegando al $93.7 \%$ en caso de hombres y de $73.7 \%$ en el caso de mujeres.

Sobre la continuidad del consumo de drogas en quienes consumieron por primera vez, el $82.8 \%$ del total de los entrevistados respondió afirmativamente. En la muestra de reclusos, el $83.1 \%$ aceptó la continuidad del consumo y en reclusas el 78.9\% respondió afirmativamente; no se encontró diferencia estadística significativa entre ambos grupos respecto a la continuidad del consumo $\left(\mathrm{X}^{2}=0.210\right.$, $p=.647$ ). De quienes aceptaron historia de consumo, el $20.4 \%$ de reclusos señaló haber asistido en algún momento a tratamiento para consumidores; el porcentaje fue de $57.9 \%$ en las reclusas; encontrándose diferencia estadística significativa entre reclusos y reclusas $\left(\mathrm{X}^{2}=14.064, p=.000\right)$.

En relación a conductas infractoras o antisociales antes de los 18 años, con o sin ingreso al sistema de justicia penal, el $56.7 \%$ del total de entrevistados refirió haberlas realizado. En el caso de reclusos, el porcentaje fue $61.1 \%$ y en el caso de las reclusas, el 18.9\%; encontrándose diferencia estadística significativa entre ambos grupos $\left(\mathrm{X}^{2}=23.997, p=.000\right)$. La edad promedio de la primera conducta infractora auto referida fue 15.21 años en reclusos y 14.71 años en reclusas, sin evidenciar diferencia significativa entre grupos $(t=0.738, p=.461)$. El 10.6\% de reclusos refirió tener ingreso a centros juveniles y el 6.2\% manifestó haber cumplido sentencia en medio abierto juvenil o Servicio de Orientación al Adolescente (SOA); en caso de las reclusas, el 5.4\% reconoció ingreso a un centro juvenil.

El 38\% del total de entrevistados refirió uso de arma de fuego en su accionar delictivo; en reclusos el porcentaje fue $38.9 \%$ y en reclusas $29.7 \%$; no se encontró diferencias significativas entre grupos $\left(x^{2}=1.195, p=.274\right)$. Respecto a uso de arma blanca en la acción delictiva, el $11.7 \%$ del total aceptó haberlas usado; en reclusos el porcentaje fue de $12.1 \%$ y en reclusas el $8.1 \%$; no se encontró diferencia estadística significativa entre hombres y mujeres $\left(\mathrm{x}^{2}=0.523, p=.469\right)$. El $93 \%$ del total de entrevistados refirió el uso de la intimidación como estrategia para el delito; el porcentaje fue de $95.6 \%$ en reclusos y $70.3 \%$ en reclusas; con diferencia significativa entre los grupos $\left(\mathrm{x}^{2}=32.608, p=.000\right)$. Se encontró, que reclusos (79\%) y reclusas $(89.3 \%)$ realizaron el delito acompañado por otras personas. 
Al indagar sobre la educación básica, el $60.8 \%$ del total no culminó la secundaria. Este porcentaje en reclusos fue de $60.60 \%$ y en reclusas $62.20 \%$. Considerando los años estudiados, el promedio fue de 9.13 años en reclusos y 9.03 años en reclusas; es decir, tercero de secundaria; no se encontró diferencia significativa entre grupos $(t=0.257, p=.798)($ ver Figura 1$)$.

Figura 1

Factores de riesgo individuales presentes en las muestras de estudio

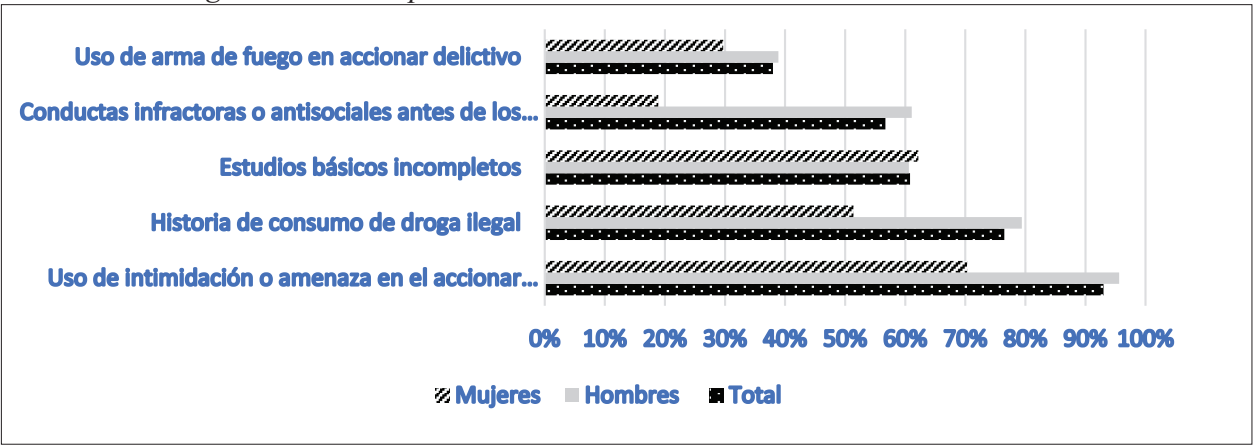

\section{Factores de riesgo relacionados con el contexto familiar:}

Dentro de los factores de riesgo del contexto familiar, el 38.3\% del total de entrevistados, refirió separación de sus padres durante la niñez. El 36.8\% de reclusos esta situación, en las reclusas esto ocurrió en el 51.4\%; estos datos no reflejaron diferencia estadística significativa entre grupos $\left(\mathrm{X}^{2}=2.990, p=.084\right)$. El $53 \%$ del total de entrevistados refirió haber fugado del hogar en una o más oportunidades durante de la infancia. Este factor se presentó en el $51 \%$ de reclusos y en el $73 \%$ de reclusas; confirmándose diferencia significativa entre ambos grupos $\left(\mathrm{X}^{2}=6.383, p=.012\right)$.

En relación a la exposición como observador de violencia en el hogar durante la niñez, el 56.1\% del total de entrevistados respondió afirmativamente. Esta situación se presentó en $54,8 \%$ de los reclusos y en el $67.6 \%$ de reclusas; no representando diferencia estadística significativa entre grupos $\left(\mathrm{X}^{2}=2.187, p=.139\right)$. Sobre violencia física y/o psicológica sufrida en el hogar en la infancia, el $45.5 \%$ del total refirió haberla sufrido. Situación reconocida por el $44,2 \%$ de reclusos y el $56,8 \%$ de reclusas; no se reflejó diferencia estadística significancia entre ambos grupos $\left(\mathrm{X}^{2}=2.097, p=.148\right)$.

Sobre familiares con conductas antisociales en general, el $62 \%$ de los entrevistados reconoció tenerlos. En reclusos, el 63\% reconoció esta situación y en reclusas, el 54.1\%; proporciones que no evidencian diferencias estadísticas significativas entre grupos $\left(\mathrm{X}^{2}=1.119 ; p=.290\right)$. En relación a familiares con ingreso a establecimientos penitenciarios o centros juveniles, el 52.5\% del total reconoció esta realidad. Este factor fue aceptado por el $53,9 \%$ de reclusos y por el $40.5 \%$ de 
reclusas; estos porcentajes no reflejaron diferencia estadística significativa entre ambos grupos $\left(\mathrm{X}^{2}=2.363, p=.124\right)$ (ver Figura 2$)$.

\section{Figura 2}

Factores de riesgo relacionados con el contexto familiar

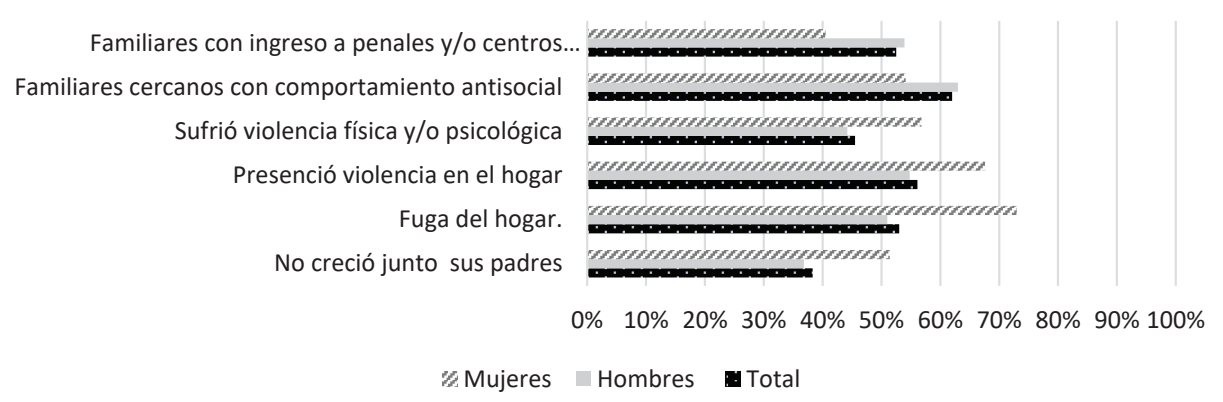

El 100\% de reclusas y reclusos señalaron tener historia laboral, aunque se refirieron a trabajos en su mayoría no calificados, informales y de baja remuneración. Al preguntar por la edad que empezaron a trabajar por primera vez, se obtuvo como edad promedio 14,9 años en el caso de hombres y 15,5 en el caso de mujeres; no representando diferencia estadística significativa $(t=-1.003, p=.317)$.

\section{Factores de riesgo relacionados con el contexto social.}

En relación a factores de riesgo provenientes del contexto social, el 95\% del total de entrevistados reconoció características negativas en su barrio o vecindario de procedencia. El porcentaje en reclusos fue $96.0 \%$ y $86.5 \%$ en reclusas, no se encontró diferencias significativas entre los grupos $\left(\mathrm{X}^{2}=6.222, p=.13\right)$. Entre las características negativas del barrio de procedencia, el 52\% del total señaló barrios desfavorecidos socio-económicamente; siendo este porcentaje de $53.0 \%$ en reclusos y $43,2 \%$ en reclusas; no se evidenció diferencia estadística significativa entre grupos $\left(\mathrm{X}^{2}=1.255, p=.263\right)$.

El $83.5 \%$ del total refirió delincuencia visible en su barrio de procedencia. En el caso de reclusos el porcentaje fue del $89.6 \%$ y de $83,8 \%$ en reclusas; estos valores no representaron diferencia estadística entre grupos $\left(\mathrm{X}^{2}=0.002, p=.964\right)$. También, el 73.5\% reconoció venta de drogas en el barrio de procedencia y/o en zonas próximas; este porcentaje fue de $73.5 \%$ en reclusos y $73.0 \%$ en reclusas; no reflejando diferencias significativas entre ambos grupos $\left(X^{2}=0.005, p=0.943\right)$.

Otra característica existente en el barrio de procedencia reconocida por $75.4 \%$ del total de encuestados fue el consumo de drogas. En reclusos el porcentaje fue de $76,3 \%$ y de $67,6 \%$ en reclusas; no se encontró diferencias significativas entre ambos grupos $\left(\mathrm{X}^{2}=1.372, p=.241\right)$. También la existencia de pandillas o grupos delictivos en el barrio fue una problemática reconocida por el $70.9 \%$ de entrevistados. En reclusos, 
el $72.6 \%$ refirió este problema y en reclusas el 56,8\%; evidenciando diferencia estadística significativa entre ambos grupos $\left(\mathrm{X}^{2}=4.033, p=.045\right)$.

El $86.3 \%$ de entrevistados reconocen tener amigos cercanos con conductas antisociales diversas. En reclusos el porcentaje fue de $87.5 \%$ y $75.7 \%$ en reclusas; encontrándose diferencia significativa entre ambos $\left(\mathrm{X}^{2}=3.952, p=.047\right)$. En relación a tener amigos cercanos con conductas delictivas activas, el 69\% del total respondió afirmativamente. El porcentaje fue de $70.1 \%$ en reclusos y de $59.5 \%$ en reclusas; sin diferencia estadística significativa entre los grupos $\left(\mathrm{X}^{2}=1.754, p=\right.$ $.185)$. El $52 \%$ del total aceptó tener amistades que venden drogas ilegales; esto se presentó en el 53.3\% de reclusos y en el $40.5 \%$ de reclusas; sin evidenciar diferencia significativa entre grupos $\left(\mathrm{X}^{2}=2.154, p=.142\right)$.

En relación a amigos que consumen drogas, el 70.9\% del total de entrevistados respondió afirmativamente. En reclusos el porcentaje fue de $73,8 \%$ y de $45.9 \%$ en reclusas; se obtuvo diferencia significativa entre grupos $\left(X^{2}=12.517\right.$, $p=.000)$. El 47,8\% del total aceptó tener amigos cercanos que usan armas de fuego para conductas ilegales; el porcentaje en reclusos fue de $48.9 \%$ y $37.8 \%$ en reclusas; sin diferencia estadística significativa entre ambos grupos $\left(\mathrm{X}^{2}=1.630\right.$, $p=.202$ ). Amigos que utilizan arma blanca fue reconocido por $29.1 \%$ del total de entrevistados; el $28,7 \%$ correspondió a reclusos y el $32.4 \%$ a reclusas; no se obtuvo diferencia estadística significativa entre grupos $\left(\mathrm{X}^{2}=0.229, p=.632\right)$.

El $74.9 \%$ del total de entrevistados refirió conocer personas, entre amigos y conocidos, con ingreso a establecimientos penitenciarios; el porcentaje fue de $91,8 \%$ en el caso de reclusos y del $59.5 \%$ en reclusas. Reflejándose una diferencia estadística significativa en esta variable $\left(\mathrm{X}^{2}=5.201, p=.023\right)$ (ver Figura 3).

\section{Figura 3}

Factores de riesgo del contexto social presentes en las muestras de estudio

Amigos y conocidos con ingreso a establecimientos... Amigos que consumen alcohol de manera frecuente Amigos que usan armas de fuego Amigos que consumen drogas Amigos que venden drogas ilegales Amigos cercanos con conducta delictiva activa Amigos cercanos con conducta antisocial Existencia de pandillas o grupos delictivos en el.. Consumo de drogas en el barrio o zonas próximas Venta de drogas en el barrio o zonas próximas Delincuencia visible en el barrio Marginalidad o barrio desfavorecido socioVecindario o barrio con caracteristicas negativas

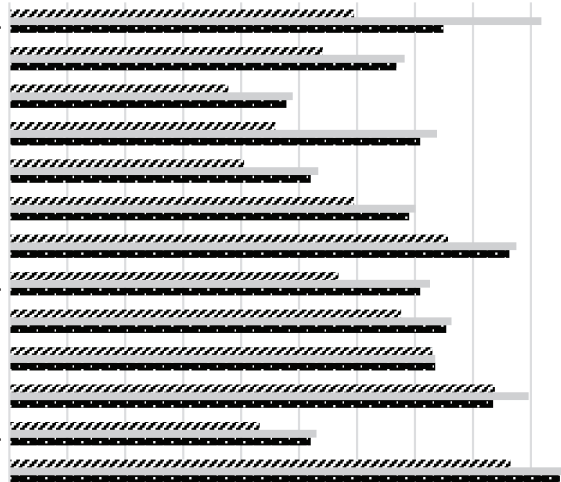

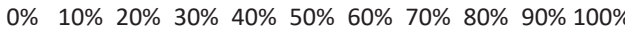

Z Mujeres Hombres Total 


\section{Acumulación de factores de riesgo}

En relación a los principales factores de riesgo recogidos en el estudio, en los ámbitos, individual (Crecer junto a ambos padres, fuga del hogar durante infancia o adolescencia, estudios básicos incompletos, consumo de droga, conducta antisocial antes de los 18 años, uso de violencia -arma de fuego o arma blanca- en el delito actual), familiar (presenciar violencia familiar, sufrir violencia en su familia durante su infancia, familiares con conducta antisocial) y social (barrio con características desfavorables o negativas, amigos cercanos o pares con conducta antisocial); el número promedio de factores acumulados en el total de entrevistados fue de 6.7. En el caso de reclusos fue de 6.8 factores y de 6.3 en el caso de reclusas; no se evidenció diferencia estadística significativa entre ambos grupos en relación al promedio de factores de riesgo encontrados en su historia de vida $(t$ $=1.213, p=.226$ ).

\section{DISCUSIÓN}

Los hallazgos empíricos del estudio confirman la existencia de múltiples factores de riesgo que la literatura especializada relaciona con la conducta delictiva y violenta, presentes en la historia de vida de reclusas y reclusos peruanos sentenciados por delitos contra el patrimonio. La historia de consumo de drogas está presente en la mayoría de ellos, aunque con mayor prevalencia en reclusos ( $79 \%$ de reclusos y $51.4 \%$ de reclusas); la principal droga de inicio es la marihuana y la edad promedio de inicio de consumo es de 15.31 años en reclusos y 16.21 años en reclusas. Además, la mayoría de entrevistados presentó continuidad de consumo.

Estos resultados coinciden con lo encontrado por Pérez y Ruiz, (2017) al señalar que el abuso de sustancias como el alcohol y la marihuana o cannabis representan un riesgo para el desarrollo de conductas delictivas; además, es consistente con lo encontrado por McBride et al. (2011) respecto a un uso importante de drogas en prisiones, comportamiento delictivo significativo entre consumidores y correlación significativa entre el uso de drogas y la delincuencia.

Con estos datos podemos confirmar que la implementación de programas de tratamiento especializado para consumidores de drogas en los establecimientos penitenciarios es imprescindible a fin de reducir la probabilidad de reincidencia delictiva. También son necesarios, la implementación de programas de prevención de consumo de drogas en zonas de riesgo social y criminológico, con el objetivo de evitar el inicio temprano de consumo en poblaciones juveniles y reducir de esta manera la acumulación de factores de riesgo relacionados con la delincuencia.

La incursión en conductas infractoras antes de los 18 años de edad, también es un factor de riesgo presente en la historia de vida de los reclusos y reclusas, independiente de si fueron denunciadas, judicializadas o no; sin embargo, esta variable presenta diferencias significativas entre hombres (61.1\%) y mujeres (18.9\%), 
diferencia que puede estar mediada por temas de tipo cultural, de mayor libertad en hombres que en mujeres durante la infancia y adolescencia. Hallazgo que concuerda con lo encontrado por Van Hazebroek et al. (2019), la infracción de inicio temprano es un factor de riesgo de la conducta delictiva persistente sobre todo en hombres y niños de vecindarios desfavorecidos, en delitos contra la propiedad, vandalismo, delitos violentos y sexuales. Coincide también con los mencionado por Loeber y Farrington (2000), los infractores de inicio temprano tienen alto riesgo de desarrollar comportamientos delictivos posteriores. Por tanto, es necesario tomar en cuenta este factor en la implementación de políticas y programas preventivos sostenidos en población juvenil, orientados a disminuir factores de riesgo, desarrollar o potenciar factores de protección e interrumpir o evitar el inicio temprano de conductas infractoras o delictivas.

Otro factor relacionado con la violencia y la acción criminal es el fácil acceso o disponibilidad ilegal a armas de fuego (Yu et al., 2020). Moore y Bergner (2016) encuentra que el aumento en la prevalencia de armas de fuego se asocia con un aumento de los delitos violentos, homicidios, violaciones, robos y asaltos. En el Perú las armas de fuego se comercializan de manera ilegal en el mercado negro y son de fácil acceso para los delincuentes en mercados como "La Cachina", "Las Malvinas", "Paruro", "Leticia", "Tacora" (Martell, 2019); el estudio encuentra que el $38.9 \%$ de hombres y el $29.7 \%$ de mujeres hizo uso este tipo de armas en su accionar delictivo; este hallazgo estaría apoyando el efecto de instrumentalidad de las armas de fuego y su rol en la agravación de los actos violentos; por tanto, la erradicación de los mercados negros y de su fácil acceso también debe ser una prioridad de las acciones de prevención.

Otra característica importante encontrada tanto en hombres (79\%) como en mujeres (89.3\%) del estudio es la realización de acciones delictivas en compañía de otros delincuentes; esto otorgaría mayor seguridad y éxito en el accionar delictivo, además enfatiza la importancia de la socialización negativa y del reforzamiento diferenciado en la conducta delictiva y en la reincidencia. Hallazgo importante si consideramos que quienes realizan conductas delictivas acompañados por otros delincuentes, tienen más probabilidad de reincidir que los que actúan solos, al menos en la juventud y adultez temprana, especialmente en delitos contra la propiedad (Andresen y Felson, 2012).

En el contexto educativo, el estudio encuentra que $60.60 \%$ de hombres y el $62.20 \%$ de mujeres no culminó la educación básica; es decir, la deserción escolar está presente de manera significativa en los delincuentes varones y mujeres. Estos datos coinciden con lo encontrado por Bäckman (2017), al describir que tasas generales de condenas son mayores entre los que abandonaron la escuela que entre los que no lo hicieron, tanto para mujeres como para hombres. No es claro si los arrestos, la delincuencia o la deserción es primero, lo claro es la relación existente entre abandono escolar y delincuencia, y que estos también pueden estar mediados 
por factores como las características familiares, individuales, de la escuela, entre otros (Rud et al., 2018). También Maynard et al. (2015) encuentra un fuerte patrón de asociaciones entre el comportamiento delictivo y el abandono de la escuela.

Es importante señalar que la familia puede ser un factor de protección, pero también puede resultar en un factor de riesgo (Argaez et al., 2018). El contexto familiar, es un sistema donde pueden generarse, potenciarse o acentuarse los primeros factores de riesgo para luego adicionarse otros en función a los subsistemas o sistemas donde la persona vaya interactuando, como el colegio, el barrio, los amigos, el trabajo, entre otros. Pero, también en él pueden amortiguarse, neutralizarse o mitigarse factores de riesgo individuales o sociales. La familia tiene un rol importante en el desarrollo integral del menor, prepara a los niños y adolescentes para el desenvolvimiento social y para su integración positiva en la sociedad.

Las interacciones positivas con los padres o cuidadores durante la infancia, generan apego seguro, la percepción de seguridad del subsistema parental constituye un mecanismo fundamental para la adaptación social del menor y para sus futuras formas de interacción social saludables (Cantón et al., 2010). En el estudio, tanto hombres $(54.8 \%)$ como mujeres $(67.6 \%)$ presenciaron violencia en su hogar durante su infancia; y otro porcentaje representativo de hombres $(44.2 \%)$ y de mujeres $(56.8 \%)$ fue víctimas de violencia por parte de padres o cuidadores. Es decir, en esta población la familia no siempre constituyó factor de protección, sino mayormente un factor de riesgo, facilitando modelos de violencia como forma de resolver problemas, afrontar situaciones estresantes o conseguir objetivos, alejándose de la generación de un apego seguro y un desarrollo emocional adaptativo. Es importante aquí lo señalado por Safranoff y Tiravassi (2018), tanto la exposición a violencia en la familia como la victimización, están relacionadas con la conducta delictiva posterior; haber presenciado violencia entre los padres afecta principalmente a las mujeres, incrementando la probabilidad de desarrollar conducta delictiva persistente y violenta; en caso de los hombres, la violencia directa o victimización representa mayor efecto sobre la futura delincuencia, reincidencia, trayectoria delictiva y violencia.

El abuso físico y psicológico en la edad infantil también aumenta el riesgo que el menor escape de casa en la adolescencia; y, este huir de casa aumenta el riesgo de delincuencia y victimización (Kim et al., 2009). En el presente estudio, el $51 \%$ de hombres y el $73 \%$ de mujeres presenta el factor de riesgo fuga de casa en una o más oportunidades durante de la infancia o adolescencia, la diferencia es significativa y de mayor afectación en mujeres. Coincidimos con Johnson et al. (2016), cuando señala que los padres siguen siendo importantes más allá de la adolescencia y el tipo de vínculo entre padres e hijos influye en la trayectoria de la conducta antisocial; por tanto, la mejora en la calidad de vínculo de los adolescentes con sus padres, se corresponde con la disminución en los niveles de comportamiento antisocial. En el estudio, la fuga del hogar resulta ser indicador importante 
de la escasa o nula efectividad de la familia como sistema protector; por el contrario, genera estímulos para orillar a los adolescentes a la calle, donde el riesgo de incursión de conductas antisociales, de victimización, de obtener reforzamiento diferenciado favorable al delito y a otras conductas antisociales, es mayor.

Otro factor de riesgo encontrado en el contexto familiar de los entrevistados, es la existencia de familiares con conductas antisociales, llámese, consumo de drogas, delincuencia o violencia, presente en hombres (63\%) y en mujeres (54.1\%). Además, familiares con ingreso a establecimientos penitenciarios o centros juveniles fue otra realidad reconocida por el $53.9 \%$ de hombres y el $40.5 \%$ de mujeres. Es muy probable que este tipo de contexto familiar incida en el inicio y mantenimiento de la conducta delictiva a través de mecanismos del aprendizaje social. Al respecto, Van de Rakt et al. (2009) encuentra que las condenas penales de miembros de la familia cercanos se relacionan efectivamente con el riesgo de conducta individual. En el mismo sentido Johnson et al. (2016) encuentra que la delincuencia de los padres se asocia con una trayectoria de comportamiento antisocial de los hijos.

La situación laboral también se representa un factor de riesgo importante, si bien todos los entrevistados refieren haber tenido historia laboral previa, es el subempleo, la informalidad y la baja remuneración las características significativas del tipo de empleo obtenido en el $100 \%$ de hombres y mujeres. Al respecto, no existe mucha investigación sobre factores de subempleo y delincuencia. Sin embargo, la relación entre crimen y desempleo es compleja. Andresen (2012) encuentra una relación negativa entre desempleo y delincuencia a corto plazo, pero positiva a largo plazo, concordante con la hipótesis de Cantor y Land (1991). Para Altindag (2012) desempleo y condiciones económicas tiene influencia positiva en delitos contra la propiedad. Podemos inferir entonces que la acción delictiva genera mayor perspectiva de ingresos económicos, de manera rápida y al parecer con baja probabilidad de sanción penal, situación que favorece la formación de autoeficacia delictiva en estos delincuentes.

La literatura también considera al contexto social como un proveedor importante de factores de riesgo para la conducta delictiva, el estudio encontró que casi la totalidad de entrevistados, entre hombres (96\%) y mujeres $(86.5 \%)$ reconocieron alguna característica negativa en su barrio o vecindario de procedencia, como marginalidad o desfavorecimiento socioeconómico, delincuencia visible, venta y consumo de drogas ilegales o existencia de grupos delictivos. Damm y Dustmann (2014) enfatizan que es la proporción de delincuentes que viven en el área y no la tasa de delitos cometidos, lo que afecta la participación delictiva individual posterior, pero señala como factor clave la interacción social y amistad con los delincuentes lo que incrementa la probabilidad de delincuencia posterior. El efecto será más importante si esta interacción social se da en la adolescencia, edad de mayor 
vulnerabilidad a la influencia de compañeros. En la edad adulta el reforzamiento y el éxito delictivo también son factores de riesgo importantes.

En el mismo sentido hay un reconocimiento o aceptación en la mayoría de entrevistados sobre amigos cercanos con conductas antisociales diversas, siendo más significativo en hombres (87.5\%) que en mujeres (75.7\%); dentro de estas se identificaron conductas delictivas, venta de drogas ilegales, consumo de drogas ilegales, uso de armas de fuego en conductas delictivas o violentas, consumo de alcohol de manera frecuente y amigos con ingreso a establecimientos penitenciarios. Al respecto, la literatura especializada identifica tener amigos delincuentes como un predictor significativo de conducta delictiva, también son significativos exposición en la calle a violencia y delincuencia (Redondo, 2015).

No hay que perder de vista que muchos de los factores analizados en el estudio se encuentran vinculados a la reincidencia delictiva y carrera criminal. Farrington (2020) considera que una disciplina dura, la supervisión deficiente de los padres, tener al padre condenado y conflicto entre padres, conforman factores de riesgo en la infancia relacionados con el desarrollo de delincuencia persistente. Whitten et al. (2019) encuentra que la separación de los padres y la hiperactividad pueden predecir de manera significativa la delincuencia persistente; en tanto, Salvador et al. (2013) afirman que la edad de inicio de consumo de drogas, los antecedentes penales y el nivel educativo se asocian significativamente con la reincidencia delictiva. También la historia de conducta antisocial, el patrón de personalidad antisocial, la asociación con compañeros antisociales y los problemas laborales son significativos en la predicción de la reincidencia delictiva (Nguyen et al., 2011; Yukhnenko et al., 2019).

\section{CONCLUSIONES}

Las personas privadas de libertad por delitos contra el patrimonio, entrevistados en el estudio, presentaron edades promedio correspondiente a adultos jóvenes y se encontraban recluidos principalmente por el delito específicos de robo agravado tanto en el caso de hombres como en el de mujeres. Presentaron de manera importante en su historia de vida, factores de riesgo de conducta delictiva en el ámbito individual, familiar y social; siendo el promedio de 6.7 factores de riesgo identificados, del total de factores indagados. No se encontró diferencias estadísticamente significativas en el número de factores de riesgo presentes en la historia de vida entre hombres y mujeres.

En el ámbito individual se identificó en los entrevistados hombres y mujeres, de manera importante, historia de consumo de drogas ilegales, inicio temprano de consumo e inicio de conductas infractoras o antisociales antes de los 18 años. También se identificó la existencia de amigos cercanos con conductas antisociales diversas, con conductas delictivas activas, amigos que venden drogas, que consumen drogas, 
amigos que usan armas de fuego, amistades cercanas que consumen alcohol de manera frecuente, amigos y conocidos con ingreso a establecimientos penitenciarios.

En el contexto familiar se encontró, en la infancia de los entrevistados, porcentajes significativos de fuga del hogar, haber sido víctima de violencia en el hogar o haber presenciado violencia intrafamiliar. También se identificó presencia significativa de familiares con conductas antisociales y familiares con ingreso a centros penitenciario o centros juveniles.

En el ámbito educativo, se identificó de manera recurrente deserción escolar en la mayoría de entrevistados, siendo el promedio de estudios realizados, 9 años o tercero de secundaria. En el contexto laboral, se identificó en los entrevistados un historial laboral de subempleo, baja remuneración e informalidad. En relación con el contexto social, se identificó de manera importante, presencia de características negativas o desfavorables en el vecindario de procedencia como son marginalidad o barrios desfavorecidos socio-económicamente, delincuencia visible, venta de drogas en el barrio de procedencia y/o en zonas próximas, consumo de drogas, existencia de pandillas o grupos delictivos.

Si bien los factores de riesgo descritos se encontraron en la mayoría de hombres y mujeres del estudio, se evidenció diferencias estadísticamente significativas en algunos factores de riesgo, según el sexo. En la historia de consumo de drogas, auto reporte de conductas infractoras o antisociales antes de los 18 años, aceptación de existencia de pandillas o grupos delictivos en el barrio, reconocimiento de amigos cercanos con conductas antisociales diversas, amigos consumidores de dogas y amigos o conocidos con ingreso a establecimientos penitenciarios, la proporción fue significativamente mayor en el caso de reclusos. En el factor de riesgo fuga del hogar en una o más oportunidades durante de la infancia, se encontró diferencia estadísticamente significativa a favor de las mujeres.

La evidencia científica demuestra que estos factores de riesgo tienen relación significativa con la conducta delictiva. Por tanto, estos datos deberían tomarse en cuenta en el diseño y ejecución de políticas y programas de prevención de la delincuencia, tomando en cuenta que la prevención temprana es más efectiva que la intervención tardía, es decir, cuando ya la trayectoria delictiva ha sido consolidada, resultando más complejo, difícil y costoso el tratamiento resocializador. También, es importante programas sociales que trabajen con los hijos e hijas menores de las personas privadas de libertad, quienes al pertenecer a los mismos contextos que sus padres, existe probabilidad de que puedan presentar similares factores de riesgo a los padres en su historia de vida.

\section{Fuentes de financiamiento}

La investigación fue autofinanciada. 


\section{Aspectos éticos}

El autor declara haber actuado éticamente según los códigos de investigación.

\section{Conflicto de intereses}

El autor declaró no tener ningún conflicto de intereses con respecto a la investigación, autoría y publicación de este artículo.

\section{Agradecimientos}

Se agradece a la Dirección de Tratamiento del Instituto Nacional Penitenciario y al voluntariado "Criminólogos en acción" y al Observatorio Nacional de Política Criminal del Ministerio de Justicia y Derechos Humanos.

\section{REFERENCIAS}

Alemu, A., Kisely, S., Williams, G., Strathearn, L., Clavarino, A., \& Najman, J. M. (2017). Gender differences in delinquency at 21 years following childhood maltreatment: A birth cohort study. Personality and Individual Differences, 106, 95-103. https://doi. org/10.1016/j.paid.2016.10.020

Altindag, D. T. (2012). Crime and unemployment: Evidence from Europe. International Review of Law and Economics, 32(1), 145-157. https://doi.org/10.1016/j.irle.2011.10.003

Andresen, M. A. (2012). Unemployment and crime: A neighborhood level panel data approach. Social Science Research, 41(6), 1615-1628. https://doi.org/10.1016/j.ssresearch.2012.07.003

Andresen, M. A., \& Felson, M. (2012). Co-Offending and the Diversification of Crime Types. International Journal of Offender Therapy and Comparative Criminology, 56(5), 811-829. https://doi.org/10.1177/0306624X11407154

Argaez, S. G. A., Echeverría, R. E., Evia, N. M. E., \& Carrillo, C. D. C. (2018). Prevención de Factores de Riesgo en Adolescentes: Intervención para Padres y Madres. Psicologia Escolar e Educacional, 22(2), 259-269. https://doi.org/10.1590/2175$\underline{35392018014279}$

Bäckman, O. (2017). High School Dropout, Resource Attainment, and Criminal Convictions. Journal of Research in Crime and Delinquency, 54(5), 715-749. https://doi.org/ doi.org/10.1177/0022427817697441

Bazdrech, M. (2001). Educación y pobreza: Una relación conflictiva. En Pobreza, desigualdad social y ciudadanía. Los límites de las políticas sociales en América Latina (pp. 65-81). CLACSO. http://bibliotecavirtual.clacso.org.ar/clacso/ gt/20101029064158/6ziccardi.pdf

Cantón, J., Cortés, M., \& Cantón-Cortés, D. (2010). APEGO, SEGURIDAD EN EL SISTEMA FAMILIAR Y ACTITUDES ANTE LA VIDA. International Journal of Developmental and Educational Psychology, 2(1), 251-258. 
Cantor, D., \& Land, K. C. (1991). Exploring Possible Temporal Relationships of Unemployment and Crime: A Comment on Hale and Sabbagh. Journal of Research in Crime and Delinquency, 28(4), 418-425. https://doi.org/10.1177/0022427891028004003

Capdevila, M., Blanch, M., Ferrer, M., Andres-Pueyo, A., Framis, B., Comas, N., Garrigós, A., \& Boldú, A. (2014). Prison recidivism rate 2014. Executive Report. 23.

Casey, S. (2011). Understanding Young Offenders: Developmental Criminology. The Open Criminology Journal, 4(1), 13-22. https://doi.org/10.2174/1874917801104010013

Damm, A. P., \& Dustmann, C. (2014). Does Growing Up in a High Crime Neighborhood Affect Youth Criminal Behavior? The American Economic Review, 104(6), 18061832.

DeLisi, M., \& Piquero, A. R. (2011). New frontiers in criminal careers research, 2000 2011: A state-of-the-art review. Journal of Criminal Justice, 39(4), 289-301. https:// doi.org/10.1016/j.jcrimjus.2011.05.001

Esbec, E., \& Echeburúa, E. (2016). Abuso de drogas y delincuencia: Consideraciones para una valoración forense integral. Adicciones, 28(1), 48-56. https://doi.org/10.20882/ adicciones.790

Estrada Pineda, C., Rodríguez Díaz, F. J., Cerros Rodríguez, E., \& Solano Mendoza, C. (2015). Implicaciones parentales en las conductas delictivas de adolescentes: Tendencias y narrativas. Papeles de población, 21(84), 107-132.

Fachelli, S. (2019). El rol de la educación superior en la movilidad ocupacional intergeneracional: Análisis comparado entre Argentina y España. Revista de Educación y Derecho, O(19), Article 19. https://doi.org/10.1344/re\&d.v0i19.28360

Farrington, D. (2006). Childhood risk factors and risk-focussed prevention. En The Oxford Handbook of Criminology (4th ed.). Oxford University Press, in press. https://citeseerx.ist.psu.edu/viewdoc/download?doi=10.1.1.123.2030\&rep=rep1\&type=pdf

Farrington, D. P. (2020). Childhood risk factors for criminal career duration: Comparisons with prevalence, onset, frequency and recidivism. Criminal Behaviour and Mental Health, 30(4), 159-171. https://doi.org/10.1002/cbm.2155

Farrington, D. P., Jolliffe, D., Loeber, R., Stouthamer-Loeber, M., \& Kalb, L. M. (2001). The concentration of offenders in families, and family criminality in the prediction of boys' delinquency. Journal of Adolescence, 24(5), 579-596. https://doi.org/10.1006/ jado.2001.0424

Felson, M., \& Cohen, L. E. (1980). Human ecology and crime: A routine activity approach. Human Ecology, 8(4), 389-406. https://doi.org/10.1007/BF01561001

Hoge, R. D., Vincent, G., Guy, L., \& Redondo, S. (2015). Predicción de riesgo y evaluación de necesidades de intervención con delincuentes jóvenes. Revista Española de Investigación Criminológica, 1(13), 40.

Hoskin, A. (2001). Armed Americans: The impact of firearm availability on national homicide rates. Justice Quarterly - JUSTICE Q, 18, 569-592. https://doi. org/10.1080/07418820100095021 
Instituto Nacional Penitenciario. (2018). Estudio integral y elaboración del programa de tratamiento individualizado del interno. Ficha psicosocial para sentenciados. INPE. Resolución Directoral Nº 179-2018-INPE/12

Johnson, W. L., Giordano, P. C., Longmore, M. A., \& Manning, W. D. (2016). Parents, Identities, and Trajectories of Antisocial Behavior from Adolescence to Young Adulthood. Journal of Developmental and Life-Course Criminology, 2(4), 442-465. https://doi.org/10.1007/s40865-016-0044-3

Kim, M. J., Tajima, E. A., Herrenkohl, T. I., \& Huang, B. (2009). Early Child Maltreatment, Runaway Youths, and Risk of Delinquency and Victimization in Adolescence: A Mediational Model. Social work research, 33(1), 19-28.

Lazarte, A. (2020). La fe religiosa de los internos del penal Sarita Colonia del Callao-Perú-Un análisis psicoanalítico | Revista EDUCA UMCH. Revista sobre Educación y Sociedad, 16(1), 5-22. https://doi.org/10.15366/Educa UMCH2019.17.3.001

Loeber, R., \& Farrington, D. (2012). From Juvenile Delinquency to Adult Crime: Criminal Careers, Justice Policy and Prevention. En From Juvenile Delinquency to Adult Crime. Oxford University Press. https:/oxford.universitypressscholarship.com/ view/10.1093/acprof:oso/9780199828166.001.0001/acprof-9780199828166

Loeber, R., \& Farrington, D. P. (2000). Young children who commit crime: Epidemiology, developmental origins, risk factors, early interventions, and policy implications. Development and Psychopathology, 12(4), 737-762. https://doi.org/10.1017/ $\underline{\mathrm{s} 0954579400004107}$

Marcelli, D., \& Braconnier, A. (2005). Manual de Psicopatologia del Adolescente (2). Masson. https://books.google.com.pe/books?id=0kmVDTRMUAgC\&printsec $=$ fron tcover\&hl $=$ es\&source $=\mathrm{gbs} \_g e \_s u m m a r y \_r \& c a d=0 \# \mathrm{v}=$ onepage $\& \mathrm{q} \& \mathrm{f}=$ false

Martell, S. (2019, marzo 21). ¿Cuánto cuesta obtener un arma de fuego en el mercado negro? RPP. https://rpp.pe/economia/economia/cuanto-cuesta-obtener-un-arma-defuego-en-el-mercado-negro-noticia-1187274

Maynard, B. R., Salas-Wright, C. P., \& Vaughn, M. G. (2015). High School Dropouts in Emerging Adulthood: Substance Use, Mental Health Problems, and Crime. Community mental health journal, 51(3), 289-299. https://doi.org/10.1007/s10597-014-9760-5

McBride, D. C., VanderWaal, C. J., \& Terry-McElrath, Y. M. (2011). The Drugs-Crime Wars: Past, Present, and Future Directions in Theory, Policy, and Program Interventions. ImpacTeen, 14, 1-116.

McGee, T. R., \& Farrington, D. P. (2019, septiembre 30). Developmental and Life-Course Theories of Crime. Oxford Research Encyclopedia of Criminology and Criminal Justice. https://doi.org/10.1093/acrefore/9780190264079.013.250

Moore, M. D., \& Bergner, C. M. (2016). The Relationship between Firearm Ownership and Violent Crime. 20.

Nguyen, T., Arbach-Lucioni, K., \& Andrés-Pueyo, A. (2011). Factores de riesgo de la reincidencia violenta en población penitenciaria. Revista de derecho penal y criminología, 6, 273-294. 
Otero, X. M. O. (1997). Droga y delincuencia: Un acercamiento a la realidad. Pirámide. https://dialnet.unirioja.es/servlet/libro?codigo $=60400$

Pérez, E., \& Ruiz, S. (2017). Substance Abuse as a Risk Factor for Criminal Behavior: A Systematic Review. Acción Psicológica, 14(2), 33-50. https://doi.org/10.5944/ ap.14.2.20748

Redondo, S. (2015). El origen de los delitos: Introducción al estudio y explicación de la criminalidad. Tirant Humanidades.

Rud, I., van Klaveren, C., Groot, W., \& van den Brink, H. M. (2018). What Drives the Relationship Between Early Criminal Involvement and School Dropout? Journal of Quantitative Criminology, 34(1), 139-166. https://doi.org/10.1007/s10940-016-9326-5

Sacks, V., Murphey, D., \& Moore, K. (2014). Adverse Childhood Experiences: National and state level prevalence. Child Trends, 28, 11.

Safranoff, A., \& Tiravassi, A. (2018). La transmisión intergeneracional de la violencia: Testimonios desde la cárcel. BID. https://publications.iadb.org/publications/spanish/ document/La-transmisi\%C3\%B3n-intergeneracional-de-la-violencia-Testimoniosdesde-la-c\%C3\%A1rcel.pdf

Salvador, M., Silvina, M., Vallejos, M., Muniello, J., \& López, P. L. (2013). Variables asociadas a la reincidencia delictiva. Psicopatología clínica, legal y forense, 13, 47-58.

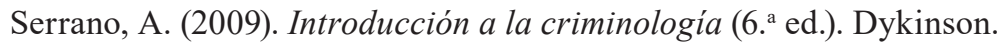

Stewart, A., Livingston, M., \& Dennison, S. (2008). Transitions and turning points: Examining the links between child maltreatment and juvenile offending. Child Abuse \& Neglect, 32(1), 51-66. https://doi.org/10.1016/j.chiabu.2007.04.011

Tanner-Smith, E. E., Wilson, S. J., \& Lipsey, M. W. (2012). Risk Factors and Crime. En The Oxford Handbook of Criminological Theory (pp. 89-111). Oxford University Press. https://doi.org/10.1093/oxfordhb/9780199747238.013.0005

Tenenbaum, G. (2016). Infracción y desistimiento: Influencias familiares en los adolescentes en conflicto con la ley de la Ciudad de Méxicol1Este artículo es parte de los contenidos de la tesis de investigación doctoral que el autor está desarrollando en el Centro de Estudios Sociológicos de El Colegio de México. Revista Mexicana de Ciencias Políticas y Sociales, 61(227), 195-221. https://doi.org/10.1016/S0185$\underline{1918(16) 30026-5}$

Thornberry, T. P., Henry, K. L., Ireland, T. O., \& Smith, C. A. (2010). The Causal Impact of Childhood-Limited Maltreatment and Adolescent Maltreatment on Early Adult Adjustment. The Journal of adolescent health : official publication of the Society for Adolescent Medicine, 46(4), 359-365. https://doi.org/10.1016/j.jadohealth.2009.09.011

Torres, G. L. M., \& Gaytán, E. A. A. (2018). Factores de riesgo asociados a la conducta violenta de los jóvenes en México. Desarrollo y Sociedad, 81, 171-210.

Uceda-Maza, F. X., \& Domínguez Alonso, J. (2017). Vinculación entre la vulnerabilidad y la exclusión social y las trayectorias delictivas. Un estudio de asociación. Psychosocial Intervention, 26(1), 29-37. https://doi.org/10.1016/j.psi.2016.07.003 
Uceda-Maza, F. X., Navarro-Pérez, J.-J., \& Pérez-Cosín, J. V. (2016). Adolescentes y drogas: Su relación con la delincuencia. Revista de Estudios Sociales, 58, 63-75. https:// doi.org/10.7440/res58.2016.05

Van de Rakt, M., Nieuwbeerta, P., \& Apel, R. (2009). Association of criminal convictions between family members: Effects of siblings, fathers and mothers. Criminal behaviour and mental health : CBMH, 19, 94-108. https://doi.org/10.1002/cbm.715

Van Hazebroek, B. C. M., Blokland, A. A. J., Wermink, H. T., De Keijser, J. W., Popma, A., \& Van Domburgh, L. (2019). Delinquent Development Among Early-Onset Offenders: Identifying and Characterizing Trajectories Based on Frequency Across Types of Offending. Criminal Justice and Behavior, 46(11), 1542-1565. https://doi. org/10.1177/0093854819876306

Vidal, S., Prince, D., Connell, C. M., Caron, C. M., Kaufman, J. S., \& Tebes, J. K. (2017). Maltreatment, family environment, and social risk factors: Determinants of the child welfare to juvenile justice transition among maltreated children and adolescents. Child Abuse \& Neglect, 63, 7-18. https://doi.org/10.1016/j.chiabu.2016.11.013

Whitten, T., McGee, T. R., Homel, R., Farrington, D. P., \& Ttofi, M. (2019). Comparing the criminal careers and childhood risk factors of persistent, chronic, and persistent-chronic offenders. Australian \& New Zealand Journal of Criminology, 52(2), 151-173. https://doi.org/10.1177/0004865818781203

Yu, S. V., Lee, D., \& Pizarro, J. M. (2020). Illegal Firearm Availability and Violence: Neighborhood-Level Analysis. Journal of Interpersonal Violence, 35(19-20), 39864012. https://doi.org/10.1177/0886260517712272

Yukhnenko, D., Blackwood, N., \& Fazel, S. (2019). Risk factors for recidivism in individuals receiving community sentences: A systematic review and meta-analysis. CNS spectrums, 25(2), 252-263. https://doi.org/10.1017/S1092852919001056 